\title{
Stage III Nasal Cavity and Paranasal Sinus Cancer AJCC v6 and v7
}

National Cancer Institute

\section{Source}

National Cancer Institute. Stage III Nasal Cavity and Paranasal Sinus Cancer A/CC v6 and v7. NCI Thesaurus. Code C67556.

Stage III includes: (T3, N0, M0); (T1, N1, M0); (T2, N1, M0); (T3, N1, M0). T3: Maxillary sinus: Tumor invading any of the following: bone of the posterior wall of maxillary sinus, subcutaneous tissues, floor or medial wall of orbit, pterygoid fossa, or ethmoid sinuses. Nasal cavity and ethmoid sinus: T umor invading the medial wall or floor of the orbit, maxillary sinus, palate, or cribriform plate. T1: Maxillary sinus: Tumor limited to the maxillary sinus mucosa with no erosion or destruction of bone. Nasal cavity and ethmoid sinus: Tumor restricted to any one subsite, with or without bony invasion. T2: Maxillary sinus: Tumor causing bone erosion or destruction including extension into the hard palate and/or middle nasal meatus, except extension to posterior wall of maxillary sinus and pterygoid plates. Nasal cavity and ethmoid sinus: Tumor invading two subsites in a single region or extending to involve an adjacent region within the nasoethmoidal complex, with or without bony invasion. N0: No regional lymph node metastasis. N1: Metastasis in a single ipsilateral lymph node, $3 \mathrm{~cm}$ or less in greatest dimension. M0: No distant metastasis. (AJCC 6th and 7th eds.) 